\title{
A REMARK ON THE IKEDA LIFT AND LOCAL SINGULAR SERIES POLYNOMIALS
}

\author{
YOUNGJU CHOIE AND WINFRIED KOHNEN
}

(Communicated by Wen-Ching Winnie Li)

\begin{abstract}
We relate a certain number-theoretic function occurring in a linear version of Ikeda's lifting map to local singular series polynomials.
\end{abstract}

\section{INTRODUCTION}

Let $k$ and $n$ be positive integers with $k \equiv n(\bmod 2)$. In his famous paper [1], T. Ikeda associated to a cuspidal Hecke eigenform $f$ of weight $2 k$ on $\Gamma_{1}:=$ $S L_{2}(\mathbf{Z})$ a cuspidal Hecke eigenform $F$ of weight $k+n$ on the Siegel modular group $\Gamma_{2 n}:=S p_{2 n}(\mathbf{Z})$ of genus $2 n$. If $n=1$, Ikeda's lifting coincides with the classical Saito-Kurokawa lift.

Let $T$ be a positive definite symmetric half-integral matrix of size $2 n$ and let $D_{T}:=(-1)^{n} \operatorname{det}(2 T)$ be its discriminant. Then $D_{T} \equiv 0,1(\bmod 4)$ and we shall write $D_{T}=D_{T, 0} f_{T}^{2}$ with $D_{T, 0}$ a fundamental discriminant and $f_{T} \in \mathbf{N}$.

Recall that the $T$-th Fourier coefficient of $F$-apart from the $\left|D_{T, 0}\right|$-th Fourier coefficient of a half-integral weight modular form - involves a product

$$
\prod_{p \mid f_{T}} \tilde{F}_{p}\left(T ; \alpha_{p}\right)
$$

where $\tilde{F}_{p}(T ; X)$ is a certain Laurent polynomial which essentially describes the "non-trivial" part of the local singular series polynomial of $T$ at $p$. Moreover, $\alpha_{p}$ is the Satake parameter of the local component $\pi_{p}$ of the automorphic cuspidal representation $\pi=\bigotimes_{p} \pi_{p}$ associated to $f$.

According to an important result of Katsurada [2], the polynomial $\tilde{F}_{p}(T ; X)$ is symmetric, i.e., is invariant under $X \mapsto X^{-1}$, and of degree $\ell_{p}:=\operatorname{ord}_{p} f_{T}$ (i.e. $X^{\ell_{p}} \tilde{F}_{p}(T ; X)$ is a polynomial of degree $\left.2 \ell_{p}\right)$. Moreover, there are certain recursion formulas that allow us to compute $\tilde{F}_{p}(T ; X)$ (knowing all the local $p$-invariants of $T)$ in an explicit, though not quite simple way [2, sect. 4].

In [5], a linear version of Ikeda's lifting map was given as a map from the "plus" space $S_{k+1 / 2}^{+}$of cusp forms of weight $k+1 / 2$ and level 4 [4] to the space $S_{k+n}\left(\Gamma_{2 n}\right)$ of cusp forms of weight $k+n$ on $\Gamma_{2 n}$. Here in the expression for the $T$-th Fourier coefficient of a lifted form, a certain somewhat mysterious, multiplicative integervalued function $\phi(a ; T)$ enters, defined for $a \mid f_{T}$. Its definition is complicated and involves a certain sum over the finitely many classes of matrices $T\left[G^{-1}\right]$, where

Received by the editors October 17, 2006 and, in revised form, April 23, 2007.

2000 Mathematics Subject Classification. Primary 11F46. 
$G$ runs through matrices in $M_{2 n}(\mathbf{Z}) \cap G L_{2 n}(\mathbf{Q})$ such that $T\left[G^{-1}\right]$ is half-integral, modulo the action of $G L_{2 n}(\mathbf{Z})$ by left-multiplication. Here we use the notation $A[B]:=B^{\prime} A B$ for matrices $A$ and $B$ of appropriate sizes. It seems quite difficult to compute $\phi(a ; T)$ explicitly from its definition, in general, and so far no more explicit and simpler expressions for $\phi(a ; T)$ (except in the case $n=1$ ) have been given in the literature.

The purpose of this paper is to relate $\phi\left(p^{j} ; T\right)\left(0 \leq j \leq \ell_{p}\right)$ in an explicit and simple way to $\tilde{F}_{p}(T ; X)$. In fact, we will show that the latter are essentially the coefficients of $\tilde{F}_{p}(T ; X)$ in a special basis of the space of symmetric Laurent polynomials of degree $\leq \ell_{p}$. In combination with the results of [2], this may lead to explicit expressions for $\phi\left(p^{j} ; T\right)\left(0 \leq j \leq \ell_{p}\right)$.

In the proof of our result several calculations and arguments given in [5], especially certain formulas for the Fourier coefficients of Siegel-Eisenstein series, play an important role.

\section{LOCAL SINGULAR SERIES}

Let $T$ be a positive definite symmetric half-integral matrix of size $2 n$. If $p$ is a prime, then the singular series attached to $T$ and $p$ is defined as

$$
b_{p}(T ; s):=\sum_{R} \nu_{p}(R)^{-s} \mathrm{e}_{p}(\operatorname{tr}(T R)) \quad(s \in \mathbf{C}),
$$

where $R$ runs over all symmetric $(2 n, 2 n)$-matrices with entries in $\mathbf{Q}_{p} / \mathbf{Z}_{p}$ and $\nu_{p}(R)$ is a power of $p$ equal to the product of denominators of elementary divisors of $R$. Furthermore, for $x \in \mathbf{Q}_{p}$ we have put $\mathrm{e}_{p}(x):=e^{2 \pi i x^{\prime}}$ where $x^{\prime}$ denotes the fractional part of $x$.

As is well-known, $b_{p}(\mathcal{T} ; s)$ is a product of two polynomials in $p^{-s}$ with coefficients in $\mathbf{Z}$. More precisely, put

$$
\gamma_{p}(T ; X):=(1-X)\left(1-p^{n}\left(\frac{D_{T, 0}}{p}\right) X\right)^{-1} \cdot \prod_{j=1}^{n}\left(1-p^{2 j} X^{2}\right) .
$$

Then

$$
b_{p}(T ; s)=\gamma_{p}\left(T ; p^{-s}\right) F_{p}\left(T ; p^{-s}\right),
$$

where $F_{p}(T ; X)$ is a certain integral polynomial with constant term equal to 1 .

If we put

$$
\tilde{F}_{p}(T ; X):=X^{-\ell_{p}} F_{p}\left(T ; p^{-n-1 / 2} X\right),
$$

then $\tilde{F}_{p}(T ; X)$ is a symmetric Laurent polynomial of degree $\ell_{p}[2]$.

\section{IKEDA'S LIFTING MAP}

Let $f$ be a normalized Hecke eigenform of weight $2 k$ on $\Gamma_{1}$. For a prime $p$ we denote by $\left\{\alpha_{p}, \alpha_{p}^{-1}\right\}$ "the" Satake $p$-parameter of $f$, so

$$
1-\lambda(p) X+p^{2 k-1} X^{2}=\left(1-p^{k-1 / 2} \alpha_{p} X\right)\left(1-p^{k-1 / 2} \alpha_{p}^{-1} X\right),
$$

where $\lambda(p)$ is the $p$-th Hecke eigenvalue of $f$ (normalized in the usual way).

Denote by $c(m)\left(m \geq 1 ;(-1)^{k} m \equiv 0,1(\bmod 4)\right)$ the Fourier coefficients of a Hecke eigenform of weight $k+1 / 2$ and level 4 in the "plus" space $S_{k+1 / 2}^{+}$corresponding to $f$ under the Shimura correspondence $[4,6]$. 
Then Ikeda's main result [1] is that

$$
F(Z):=\sum_{T>0}\left(c\left(\left|D_{T, 0}\right|\right) f_{T}^{k-1 / 2} \prod_{p \mid f_{T}} \tilde{F}_{p}\left(T ; \alpha_{p}\right)\right) e^{2 \pi i \operatorname{tr}(T Z)}
$$

is a Hecke eigenform in $S_{k+n}\left(\Gamma_{2 n}\right)$. Here $Z$ is the Siegel upper half-space of genus $2 n$ and $T$ runs over all positive definite symmertic half-integral matric es of size $2 n$.

We define the function $\phi(a ; T)\left(a \mid f_{T}\right)$ as follows [5]. First, for a prime $p$ and an integer $j \geq 0$ define $\rho_{T}\left(p^{j}\right)$ as the coefficient of $X^{j}$ in $\left(1-X^{2}\right) H_{n, p}(T ; X)$ where $H_{n, p}(T ; X)$ is the "Kitaoka's polynomial" [3], a certain integral polynomial depending on the local $p$-invariants of $T$ (for a precise definition we refer, e.g., to $(6)$, sect. 3 in [5]).

We extend the function $\rho_{T}$ multiplicatively to the whole of $\mathbf{N}$ by defining

$$
\sum_{a \geq 1} \rho_{T}(a) a^{-s}:=\prod_{p \mid f_{T}}\left(\left(1-p^{-2 s}\right) H_{n, p}\left(T ; p^{-s}\right)\right) .
$$

Finally, for $a \in \mathbf{N}$ with $a \mid f_{T}$ we set

$$
\phi(a ; T):=a^{1 / 2} \sum_{d^{2} \mid a} \sum_{G \in \mathcal{D}(T),|\operatorname{det}(G)|=d} \rho_{T\left[G^{-1}\right]}\left(\frac{a}{d^{2}}\right) .
$$

Here

$$
\mathcal{D}(T):=G L_{2 n}(\mathbf{Z}) \backslash\left\{G \in M_{2 n}(\mathbf{Z}) \cap G L_{2 n}(\mathbf{Q}) \mid T\left[G^{-1}\right] \text { half-integral }\right\},
$$

where $G L_{2 n}(\mathbf{Z})$ operates by left-multiplication. It follows from the definitions that $\phi(a ; T)$ is an integer and $a \mapsto \phi(a ; T)$ is multiplicative (cf., e.g., Remarks (ii)) after the Corollary to Theorem 1 in [5]).

The main result of [5] then states that the $T$-th Fourier coefficient of the Ikeda lift $F$ of $f$ is given by

$$
\sum_{a \mid f_{T}} a^{k-1} \phi(a ; T) c\left(\left|D_{T, 0}\right|\left(\frac{f_{T}}{a}\right)^{2}\right) .
$$

As a consequence, (1) defines a linear map from $S_{k+1 / 2}^{+}$to $S_{k+n}\left(\Gamma_{2 n}\right)$ which on Hecke eigenforms coincides with Ikeda's lift.

\section{Main Result AND PROOF}

For a non-negative integer $\ell$ we denote by $V_{\ell}$ the $(\ell+1)$-dimensional complex vector space of symmetric Laurent polynomials

$$
\sum_{\nu=0}^{\ell} c_{\nu}\left(X^{\nu}+X^{-\nu}\right) \quad\left(c_{\nu} \in \mathbf{C}\right)
$$

of degree $\leq \ell$.

For $j \in\{0,1, \ldots, \ell+1\}$ we set

$$
\psi_{j}(X):=\frac{X^{j}-X^{-j}}{X-X^{-1}} .
$$

Lemma 1. Let $\epsilon \in\{0, \pm 1\}$. Then the Laurent polynomials

$$
\psi_{j}(X)-\epsilon p^{-1 / 2} \psi_{j-1}(X) \quad(j=1, \ldots, \ell+1)
$$

form a basis of $V_{\ell}$. 
Proof. It is clearly sufficient to show that the polynomials $\psi_{j}(X)(j=1, \ldots, \ell+1)$ form a basis of $V_{\ell}$, and this is obvious because of the formula

$$
\psi_{j}(X)=X^{j-1}+X^{j-3}+\cdots+X^{-j+3}+X^{-j+1} .
$$

Theorem. Let $T$ be a positive definite symmetric half-integral matrix of size $2 n$. Let $p$ be a prime with $p \mid f_{T}$ and let $\ell=\ell_{p}:=\operatorname{ord}_{p} f_{T}$. Then

$$
\tilde{F}_{p}(T ; X)=\sum_{j=1}^{\ell+1} \phi\left(p^{\ell-j+1} ; T\right) p^{-\frac{\ell-j+1}{2}}\left(\psi_{j}(X)-\left(\frac{D_{T, 0}}{p}\right) p^{-1 / 2} \psi_{j-1}(X)\right) .
$$

Proof. Let us write $D_{0}=D_{T, 0}$ and $f=f_{T}$ (this will not lead to confusion with earlier notation). According to Siegel, the $T$-th Fourier coefficient of the SiegelEisenstein series of weight $k>2 n+1$ on $\Gamma_{2 n}$ is given by

$$
\begin{aligned}
a_{k, 2 n}(T)= & \frac{2^{n}}{\zeta(1-k) \prod_{j=1}^{n} \zeta(1-2 k+2 j)} \cdot L\left(1-k+n,\left(\frac{D_{0}}{\cdot}\right)\right) \cdot f^{k-n-\frac{1}{2}} \\
& \cdot \prod_{p \mid f} \tilde{F}_{p}\left(T ; p^{n+\frac{1}{2}-k}\right) ;
\end{aligned}
$$

cf. (10) in sect. 3 of [5]. By Theorem 2 in [5], we also have

$$
a_{k, 2 n}(T)=\frac{2^{n}}{\zeta(1-k) \prod_{j=1}^{n} \zeta(1-2 k+2 j)} \sum_{a \mid f} a^{k-n-1} \phi(a ; T) H\left(k-n,\left|D_{0}\right|\left(\frac{f}{a}\right)^{2}\right),
$$

where

$$
H\left(k,\left|D_{0}\right| m^{2}\right):=L\left(1-k,\left(\frac{D_{0}}{\cdot}\right)\right) \sum_{d \mid m} \mu(d)\left(\frac{D_{0}}{d}\right) d^{k-1} \sigma_{2 k-1}\left(\frac{m}{d}\right) \quad(m \in \mathbf{N})
$$

is Cohen's class number function. (Recall that $\sigma_{2 k-1}(a):=\sum_{d \mid a} d^{2 k-1}(a \in \mathbf{N})$.)

Combining (2) and (3), replacing $k$ by $k+n$, and using multiplicativity, we find that for all positive even integers $k$ large enough one has

$$
\begin{aligned}
\prod_{p \mid f} p^{\ell_{p}\left(k-\frac{1}{2}\right)} \tilde{F}_{p}\left(T ; p^{-k+\frac{1}{2}}\right)= & \prod_{p \mid f}\left(\sum _ { j = 0 } ^ { \ell _ { p } } p ^ { j ( k - \frac { 1 } { 2 } ) } \cdot p ^ { - \frac { j } { 2 } } \phi ( p ^ { j } ; T ) \left(\sigma_{2 k-1}\left(p^{\ell_{p}-j}\right)\right.\right. \\
& \left.\left.-\left(\frac{D_{0}}{p}\right) p^{k-\frac{1}{2}} \cdot p^{-\frac{1}{2}} \sigma_{2 k-1}\left(p^{\ell_{p}-j-1}\right)\right)\right) .
\end{aligned}
$$

We observe that

$$
\sigma_{2 k-1}\left(p^{\nu}\right)=\frac{\left(p^{k-\frac{1}{2}}\right)^{2 \nu+2}-1}{\left(p^{k-\frac{1}{2}}\right)^{2}-1} \quad\left(\nu \in \mathbf{N}_{0}\right) \ell
$$

and enter (5) with $\nu=\ell_{p}-j, \ell_{p}-j-1$ into (4).

Then by a well-known argument we deduce that identity $(4)$ holds with $p^{k-\frac{1}{2}}$ replaced by a variable $X_{p}$, for each $p \mid f$, and then it follows that

(6) $X^{\ell} \tilde{F}_{p}(T ; X)=\sum_{j=0}^{\ell} p^{-\frac{j}{2}} \phi\left(p^{j} ; T\right) X^{j}\left(\frac{X^{2(\ell-j+1)}-1}{X^{2}-1}-\left(\frac{D_{0}}{p}\right) p^{-\frac{1}{2}} X \frac{X^{2(\ell-j)}-1}{X^{2}-1}\right)$,

for each $p \mid f$ individually (with the abbreviation $\ell=\ell_{p}$ ), since the polynomials on both sides of (6) have coefficients at the highest terms equal to 1. 
Clearly, (6) can be rewritten as

$$
\tilde{F}_{p}(T ; X)=\sum_{j=0}^{\ell} p^{-\frac{j}{2}} \phi\left(p^{j} ; T\right)\left(\frac{X^{\ell-j+1}-X^{-\ell+j-1}}{X-X^{-1}}-\left(\frac{D_{0}}{p}\right) p^{-\frac{1}{2}} \frac{X^{\ell-j}-X^{-\ell+j}}{X-X^{-1}}\right),
$$

and therefore it is equivalent to the assertion of the theorem.

\section{ACKNOWLEDGMents}

This work was partially supported by KOSEF R01-2003-00011596-0 and ITRC 2006-C1090-0603-0026.

\section{REFERENCES}

[1] T. Ikeda: On the lifting of elliptic modular forms to Siegel cusp forms of degree $2 n$. Ann. of Math. (2) 154, 641-681 (2001). MR1884618 (2002m:11037)

[2] H. Katsurada: An explicit form for Siegel series. Amer. J. Math. 121 (1999), 415-452. MR1680317 (2000a:11068)

[3] Y. Kitaoka: Dirichlet series in the theory of Siegel modular forms. Nagoya Math. J. 95 (1984), 73-84. MR759464 (86b:11038)

[4] W. Kohnen: Modular forms of half-integral weight on $\Gamma_{0}(4)$. Math. Ann. 248 (1980), 249-266. MR575942 (81j:10030)

[5] W. Kohnen: Lifting modular forms of half-integral weight to Siegel modular forms of even genus. Math. Ann. 322, 787-809 (2002). MR1905104 (2003d:11067)

[6] G. Shimura: On modular forms of half-integral weight. Ann. of Math. 97 (1973), 440-481. MR0332663 (48:10989)

Department of Mathematics, Pohang Institute of Science and Technology, Pohang 790-784, KOREA

E-mail address: yjc@postech.ac.kr

Universität Heidelberg, Mathematisches Institut, INF 288, D-69120 Heidelberg, GerMANY

E-mail address: winfried@mathi.uni-heidelberg.de 